\title{
Tendencia del desarrollo puberal en escolares de la Región Metropolitana de Chile: Menor edad de presentación, mayor duración y dimorfismo sexual en la estatura
}

\author{
RAQUEL BURROWS A., XIMENA CEBALLOS S. ${ }^{a}$, \\ MEDARDO BURGUEÑO M., SANTIAGO MUZZO B.
}

\section{Trends in puberal development of school age children living in the Metropolitan Region of Chile}

Background: There is a worldwide tendency towards an earlier appearance of puberal development among children, associated with an increase in weight and height. Aim: To study the trends in puberal development in Chilean school age children, between the years 1986 and 2001 and correlate it with weight and height changes. Subjects and Methods: In two representative samples of school age children, collected between years 1985 and 1987 (m-1986) and another between years 2000 and 2002 ( $m$-2001), girls between 7 and 15 years (958 and 935, respectively) and boys between 9 and 15 years (842 and 870 respectively), were selected. Breast development (B) in females and genital development $(G)$ in males were classified according to Tanner stages. Weight, height, body mass index (BMI) and nutritional status (according to Centers for Disease Control/ National Center for Health Statistics (CDC/NCHS) standards) were assessed. Results: The prevalence of obesity increased four fold between 1986 and 2001. The 2001 generation had a significantly higher degree of puberal development than their counterparts studied in 1986. Compared to m-1986, m-2001 subjects had a lower mean age at puberal development stage 2 and 3, but no differences at puberal stages 4 and 5. BMI of m-2001 subjects was significantly higher than that of m-1986 subjects at all puberal stages. The m-2001 males showed highest stature than m-1986 in all puberal stage, however, in females there is no difference in height between m-2001 and m-1986. Conclusions: The highest BMI observed in the cohort of 2001, could be facilitating an earlier puberal development and ethnic factors could explain the sexual dimorphism in stature.

(Rev Med Chile 2010; 138: 61-67).

Key words: Body mass index; Mammary glands, human; Puberty.

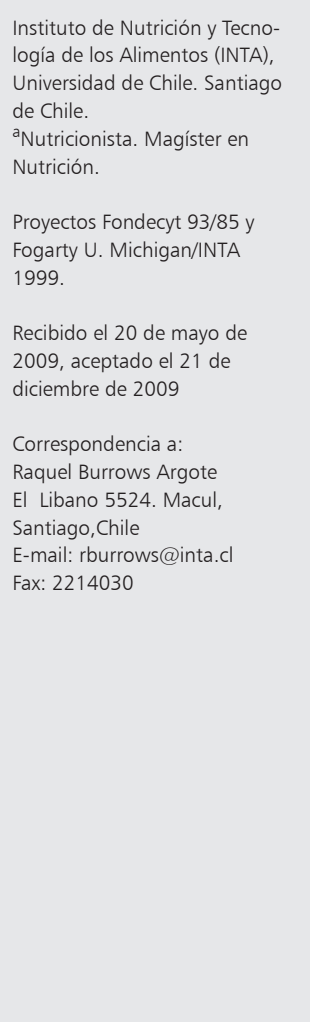

E 1 aumento progresivo en la secreción del factor liberador de gonadotropinas $(\mathrm{GnRH})$, activa el eje hipotálamo-hipófisis-gonadal (HHG) con los consecuentes cambios físicos, fisiológicos y del comportamiento propio de la pubertad $^{1}$. Este eje está bajo la influencia de factores genéticos, étnicos, nutricionales y ambientales, lo que explicaría la gran variabilidad en el inicio y progreso de la pubertad entre poblaciones contemporáneas, así como las tendencias seculares ${ }^{2,3}$. A nivel mundial, hay una tendencia a un mayor peso y estatura y a una presentación más temprana de la pubertad y menarquia, con cambios significativos durante el siglo XX. ${ }^{4-6}$ La edad de la menarquia era 
de 17 a 18 años a mediados del siglo XIX, disminuyó a 14 años a comienzo del siglo XX, llegando a los 12 años 6 meses a mediados del siglo, como consecuencia de una mejoría en la nutrición y en las condiciones de vida ${ }^{2,6}$.

Entre los factores genéticos, las migraciones y mezclas raciales y entre los ambientales, la nutrición, la actividad física y disruptores endocrinos, tendrían un rol importante en la partida más temprana de la pubertad de los últimos $60 \mathrm{años}^{2,7-9}$. Diferentes estudios coinciden en esta tendencia, así como en su asociación con el sobrepeso especialmente en las mujeres; sin embargo, difieren en la duración de la pubertad ${ }^{2,4}$. En Estados Unidos de Norteamérica (EEUU), hay una tendencia a una menor edad de partida de la pubertad con una estabilización en la edad de la menarquia y por ende, un mayor tiempo de duración de los eventos puberales ${ }^{4}$. En varios países europeos también se observa una edad más temprana de pubertad asociada al sobrepeso, pero con una menor duración ${ }^{2}$. El estudio de Bogalusa (Bogalusa Heart Study) muestra que la menor edad de menarquia en las norteamericanas se asocia a un mayor IMC, aunque habrían también determinantes étnicos, que explicarían la menor edad de partida de pubertad en las niñas de raza negra ${ }^{10,11}$. En los varones, la relación con el peso corporal se ha descrito en forma inversa ${ }^{12}$.

El primer estudio chileno sobre pubertad lo realizó la Dra. Eloisa Díaz en el año $1886^{13}$. Investigó la edad de la menarquia en 4.600 mujeres entre los 8 y los 21 años, observando que en el norte de Chile, la menarquia se presentaba mayoritariamente entre los 12 y los 14 años, en la zona central entre los 13 y los 16 años y en el sur, entre los 14 y los 16 años. Concluyó que factores climáticos y nutricionales, podrían explicar la menarquia más temprana de la mujer nortina. Estudios muy posteriores, no muestran grandes variaciones de la pubertad entre las décadas 1970-79 y 1980-89. Así, en 1974 Avendaño y cols, encontraron una edad promedio de 10,5 años para el botón mamario y de 12,4 años para la menarquia y en 1988, Burrows y Muzzo estimaron un promedio de 10,4 años y 12,4 años, respectivamente, en mujeres de similar nivel socioeconómico (NSE) ${ }^{14-16}$. Tres estudios nacionales muestran un significativo adelanto en la edad de partida de la pubertad en las mujeres, con poca variación en la edad de menarquia con las generaciones del 70 y $80^{17-19}$. Gaete y cols, encontraron $14 \%$ de botón mamario y Codner y cols $17 \%$ antes de los 8 años, con una edad de menarquia de 12,7 años. Dos de estos estudios difieren, sin embargo, en la relación entre la edad de menarquia y el peso corporal ${ }^{18,19}$. Es probable que los 3 estudios, aunque contemporáneos, no sean comparables, pues mientras uno incluyó sólo niños de NSE medio-bajo y de peso normal, los otros incluyeron niños de diferentes NSE y estado nutricional.

En Chile, la obesidad se cuadruplicó entre los años 1985 y 2000, asociada a un cambio en el perfil epidemiológico, en la dieta y en la actividad física, relacionados con un desarrollo socioeconómico "explosivo" $20-22$. Nos interesó analizar en dos muestras de escolares de similar NSE y evaluada por los mismos examinadores, los cambios seculares del desarrollo puberal durante este período de transición económica y epidemiológica tan peculiar y relacionarlo con las variaciones del crecimiento pondoestatural.

\section{Sujetos y Método}

De 2 muestras representativas, transversales y aleatorias de escolares de ambos sexos recolectadas entre los años 1985 y 1987 (m-1986) y entre los años 2000 y 2002 (m-2001), en los mismos establecimientos subvencionados (6) de la Región Metropolitana, se seleccionaron las niñas entre $7 \mathrm{y}$ 15 años (958 y 935 respectivamente) y los varones entre 9 y 16 años ( 842 y 870 respectivamente) por ser los rangos de edades donde la población normal presenta los eventos puberales. Previo al examen físico, sus apoderados firmaron un consentimiento aprobado por el Comité de Ética del INTA, a cada niño se le explicó en qué consistía este examen, el que se realizó con su consentimiento.

Antropometría y desarrollo puberal. El peso y la estatura se midieron con una balanza de precisión con cartabón (SECA) con sensibilidad de 100 g y $0,1 \mathrm{~cm}$, respectivamente, y se calculó en cada niño el IMC (Peso/Talla $\left.{ }^{2}\right)$. Los mismos pediatras (RB, MB y SM) evaluaron el desarrollo mamario en las niñas y el genital en los niños por examen visual y palpatorio y lo calificaron según los 5 estadios de Tanner ${ }^{23,24}$. El estado nutricional se evaluó utilizando el referente CDC/2000 y considerando el grado de pubertad alcanzado ${ }^{25}$. En las mujeres, se registró la fecha de presentación de la primera 
menstruación, sólo en aquellas que recordaban el año y el mes (350 de 371 en la M-1986 y 380 de 403 en la M-2001).

Estadística. Los resultados fueron procesados en planilla EXCEL y en STATA 9.0. El peso, la estatura y el IMC se expresaron como promedio y desviación estándar. Por método de Probito se calculó el promedio de edad en cada etapa de Tanner y el de la menarquia, por Chi 2 se evaluó la asociación entre la generación con el estado nutricional y el grado de pubertad alcanzado y por test de Willcoxon (Mann-Whitney) se compararon los promedios de edad, IMC y estatura dentro de una misma etapa puberal y la edad de menarquia, entre ambas generaciones. Se consideró significativo un $\mathrm{p}<0,05$.

\section{Resultados}

La Tabla 1 muestra la distribución de la muestra por edad, sexo y generación. En ambos sexos, la prevalencia de sobrepeso (Figura 1) fue mayor $(\mathrm{p}<0,0001)$ en la m-2001, que en la m-1986. En

Tabla 1. Distribución de las muestras m-1986 y m-2001 según sexo y edad

\begin{tabular}{|c|c|c|c|c|c|c|c|c|}
\hline \multirow[b]{3}{*}{ Edad (años) } & \multicolumn{4}{|c|}{ Mujeres } & \multicolumn{4}{|c|}{ Varones } \\
\hline & \multicolumn{2}{|c|}{ m-1986 } & \multicolumn{2}{|c|}{ m-2001 } & \multicolumn{2}{|c|}{ m-1986 } & \multicolumn{2}{|c|}{$m-2001$} \\
\hline & $\mathbf{N}$ & $\%$ & $\mathbf{N}$ & $\%$ & $\mathbf{N}$ & $\%$ & $\mathbf{N}$ & $\%$ \\
\hline 7 & 107 & 11,2 & 92 & 9,8 & & & & \\
\hline 8 & 106 & 11,1 & 108 & 11,6 & & & & \\
\hline 9 & 106 & 11,1 & 100 & 10,7 & 105 & 12,5 & 101 & 11,6 \\
\hline 10 & 107 & 11,2 & 106 & 11,3 & 103 & 12,3 & 119 & 13,7 \\
\hline 11 & 109 & 11,4 & 103 & 11,0 & 101 & 12,0 & 119 & 13,7 \\
\hline 12 & 112 & 11,7 & 107 & 11,4 & 97 & 11,5 & 117 & 13,4 \\
\hline 13 & 111 & 11,6 & 106 & 11,3 & 103 & 12,2 & 108 & 12,4 \\
\hline 14 & 108 & 11,3 & 99 & 10,6 & 113 & 13,4 & 108 & 12,4 \\
\hline 15 & 92 & 9,6 & 114 & 12,2 & 114 & 13,5 & 101 & 11,8 \\
\hline 16 & & & & & 106 & 12,6 & 97 & 11,1 \\
\hline Total & 958 & 100,0 & 935 & 100,0 & 842 & 100,0 & 870 & 100,0 \\
\hline
\end{tabular}

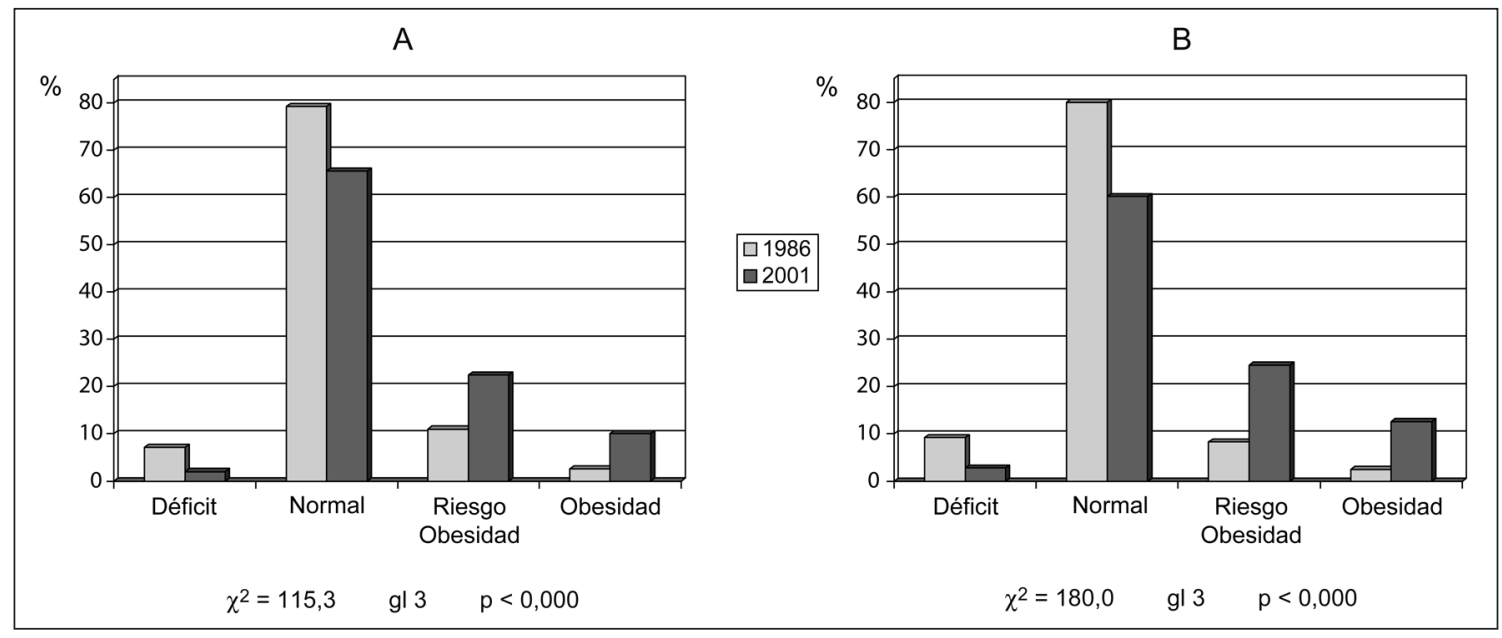

Figura 1. Distribución de las mujeres (A) y los varones (B) por estado nutricional según generación 


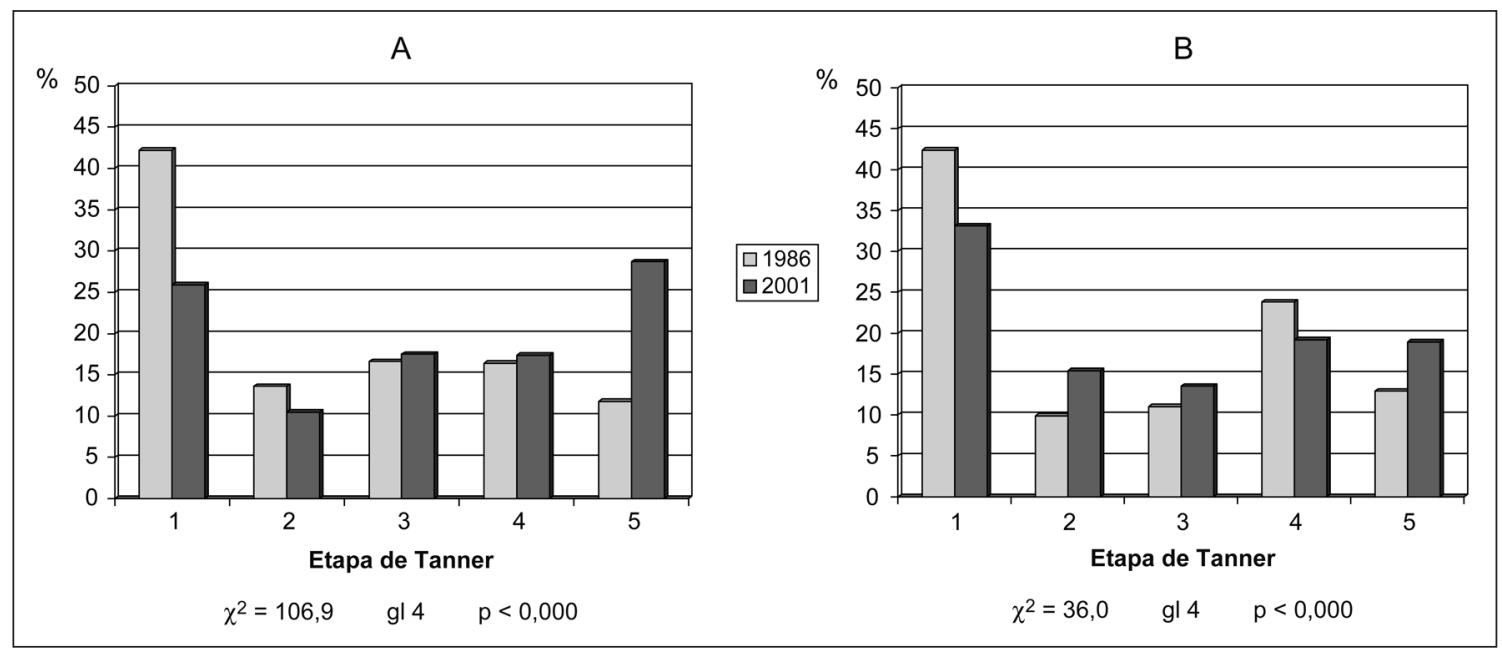

Figura 2. Distribución de las mujeres (A) y los varones (B) por estadio de Tanner según generación

Tabla 2. Edad, talla e IMC (promedio \pm DE) de las mujeres de la m-1986 y m-2001, según el estadio de Tanner

\begin{tabular}{|c|c|c|c|c|}
\hline Tanner & $\mathbf{N}$ & Edad (años) & Talla (m) & IMC \\
\hline $1(m-1986)$ & 404 & $8,9 \pm 1,3$ & $1,28 \pm 0,07$ & $16,3 \pm 2,0$ \\
\hline $1(m-2001)$ & 242 & $8,4 \pm 1,1 * *$ & $1,28 \pm 0,07$ & $17,9 \pm 2,7^{* *}$ \\
\hline $2(m-1986)$ & 125 & $10,7 \pm 1,0$ & $1,42 \pm 0,08$ & $17,7 \pm 2,5$ \\
\hline $2(m-2001)$ & 98 & $10,1 \pm 1,1 * *$ & $1,38 \pm 0,07^{* *}$ & $18,3 \pm 3,1^{* *}$ \\
\hline $3(m-1986)$ & 159 & $11,5 \pm 1,3$ & $1,46 \pm 0,08$ & $18,7 \pm 2,6$ \\
\hline $3(m-2001)$ & 164 & $10,7 \pm 1,2^{* *}$ & $1,42 \pm 0,07^{* *}$ & $20,1 \pm 2,5^{* *}$ \\
\hline $4(m-1986)$ & 157 & $13,5 \pm 1,2$ & $1,53 \pm 0,05$ & $20,1 \pm 2,5$ \\
\hline $4(m-2001)$ & 163 & $12,9 \pm 1,3^{* *}$ & $1,53 \pm 0,06$ & $21,0 \pm 3,2^{*}$ \\
\hline $5(m-1986)$ & 113 & $14,5 \pm 0,9$ & $1,58 \pm 0,06$ & $20,6 \pm 2,6$ \\
\hline $5(m-2001)$ & 268 & $14,3 \pm 1,1$ & $1,58 \pm 0,06$ & $22,4 \pm 3,1^{* *}$ \\
\hline
\end{tabular}

Significación según test de Willcoxon: ${ }^{*} p<0,001 ;{ }^{* *} p<0,000$

Tabla 3. Edad, peso, talla e IMC (promedio \pm DE) de los varones de la m-1986 y m-2001, según el estadio de Tanner

\begin{tabular}{|crlll|}
\hline Tanner & N & Edad (años) & \multicolumn{1}{c}{ Talla (m) } & \multicolumn{1}{c|}{ IMC } \\
1 (m-1986) & 357 & $10,7 \pm 1,1$ & $1,37 \pm 0,07$ & $17,2 \pm 2,4$ \\
1 (m-2001) & 288 & $10,4 \pm 1,0^{* *}$ & $1,39 \pm 0,06^{* *}$ & $19,6 \pm 3,1^{* * *}$ \\
2 (m-1986) & 83 & $12,8 \pm 1,2$ & $1,48 \pm 0,07$ & $18,5 \pm 3,2$ \\
2 (m-2001) & 134 & $12,1 \pm 0,8^{* *}$ & $1,48 \pm 0,05$ & $20,1 \pm 3,4^{* * *}$ \\
3 (m-1986) & 93 & $14,2 \pm 1,1$ & $1,58 \pm 0,07$ & $18,3 \pm 2,2$ \\
$3(m-2001)$ & 117 & $13,1 \pm 1,0^{* * *}$ & $1,57 \pm 0,06$ & $20,9 \pm 3,2^{* * *}$ \\
$4(m-1986)$ & 201 & $14,9 \pm 1,0$ & $1,63 \pm 0,07$ & $19,2 \pm 2,1$ \\
$4(m-2001)$ & 167 & $14,9 \pm 1,1$ & $66 \pm 0,06^{* *}$ & $21,0 \pm 3,2^{* *}$ \\
5 (m-1986) & 109 & $15,6 \pm 1,1$ & $1,66 \pm 0,06$ & $19,9 \pm 2,3$ \\
5 (m-2001) & 164 & $15,4 \pm 1,1$ & $1,71 \pm 0,06^{* * *}$ & $21,8 \pm 3,2^{* * *}$ \\
\hline
\end{tabular}

Significación según test de Willcoxon: ${ }^{*} p<0,001 ;{ }^{*} p<0,000$ las mujeres, el riesgo de obesidad y la obesidad alcanzaron $22,4 \%$ y $10 \%$ respectivamente en la $\mathrm{m} 2001$, vs $11,0 \%$ y $2,6 \%$ respectivamente en la $\mathrm{m}-1986$ (1A). En los varones, estos porcentajes fueron $24,5 \%$ y $12,6 \%$ respectivamente en la $\mathrm{m}-2001$ y $8,3 \%$ y $2,5 \%$ respectivamente en la m-1986 (1B).

En ambos sexos hubo una asociación $(p<0,001)$ entre el grado de pubertad y la generación (Figura 2). En las mujeres, 42,2\% de la m-1986 no presentó desarrollo mamario (M1) y $11,8 \%$ ya lo completó (M5), en cambio en la m-2001, 25,9\% estaba en M1 y 28,7\% en M5 (2A). En los varones de la m-1986, $42,2 \%$ no presentó desarrollo puberal y $12,9 \%$ ya lo completó vs $33,1 \%$ y $18,9 \%$ respectivamente en la m-2001 (2B).

La Tabla 2 muestra en las mujeres, los promedios de edad, talla e IMC según estadio de Tanner y generación. La m-2001 tiene un menor promedio de edad ( $\mathrm{p}<0,001)$ hasta la etapa 4 , sin diferencias en M5, mayor IMC en todas 
las etapas y menor estatura hasta la etapa 3 , sin diferencias en la estatura en M4 y 5, al compararla con la m-1986. La edad promedio de la menarquia, fue de $12,3 \pm 1,3$ años en la m-2001 y de 12,1 \pm 1,2 años en la m-1986, diferencia estadísticamente significativa $(\mathrm{p}<0,025)$.

En los varones (Tabla 3), la m-2001 mostró menor promedio de edad hasta G3, mayor IMC en todas las etapas puberales y mayor promedio de estatura en las etapas G1, G4 y G5, al compararlos con la m-1986.

\section{Discusión}

El objetivo de este trabajo fue investigar la tendencia de la pubertad entre los años 1985 y 2002, período en el que ocurrieron cambios importantes en los estilos de vida y en el perfil nutricional y epidemiológico de la población chilena ${ }^{19-21,26,27}$. Este período, reconocido como de post-transición, se caracteriza por un aumento significativo en la prevalencia de obesidad, cáncer, enfermedades cardiovasculares y diabetes $2^{27}$. Es interesante señalar que las patologías propias de la post transición están incluidas dentro de las consecuencias de una pubertad temprana ${ }^{28}$. En ambos sexos hay un adelanto significativo en la partida de la pubertad, sin variaciones en la edad en que se completa y un discreto adelanto de la menarquia, confirmando lo estimado por otros estudios nacionales contemporáneos $(2001 \text { y 2003 })^{17,18}$. Nuestro estudio muestra diferencias tanto en el promedio de edad $(10,1$ años) como en el porcentaje de niñas de 7 y 8 años con botón mamario (11\%), con lo encontrado por los otros autores ( 8,9 años y $25 \%$ respectivamente). Se ha planteado una sobreestimación del desarrollo mamario en niñas pequeñas con obesidad, señalándose la necesidad de desarrollar métodos que permitan diferenciar grasa de tejido mamario especialmente en el momento de la partida de la pubertad ${ }^{4}$.

La tendencia a una menor edad de presentación de la pubertad es mundial y estaría asociada al sobrepeso, a factores étnicos, al retardo del crecimiento intrauterino y a otros disruptores endocrinos ambientales ${ }^{2,4,9,29}$. Nuestro estudio muestra que la obesidad se cuadruplicó entre los años 1986 y 2001, tendencia observada en otros estudios nacionales en escolares de diferentes $\mathrm{NSE}^{20,21}$. En ambos sexos, los escolares de la m-2001 tienen
IMC significativamente mayor que la m-1986 en todas las etapas puberales. Varios estudios muestran una asociación inversa entre la adiposidad y la edad de partida de la pubertad, especialmente en las mujeres. Un seguimiento longitudinal en niñas americanas muestra que el sobrepeso a los 5 años de edad se asocia a un comienzo más temprano de la pubertad ${ }^{30}$. Otro estudio, en niñas de 6 a 9 años, muestra un z IMC significativamente mayor en las púberes que en las prepúberes; sin embargo, al controlar el peso, hay una menor edad de comienzo de la pubertad en las niñas negras, por lo que factores étnicos o ambientales desconocidos, podrían estar dando cuenta de estas diferencias ${ }^{11}$. Algunos estudios señalan que en las mujeres esta asociación sería muy fuerte, pero no en los varones. En niños norteamericanos, las mujeres de mayor peso corporal tienen menor edad de inicio puberal, pero en varones se observa lo contrario, comenzando en edad más temprana en los más delgados ${ }^{12}$. Otros estudios que investigan la relación entre grasa corporal y comienzo de la pubertad, coincide en este dimorfismo ${ }^{31}$. Esta diferencia se explicaría porque en la mujer los estrógenos promoverían un mayor desarrollo del tejido graso, mientras que en los varones, la testosterona influiría más en el crecimiento y menos en la formación de grasa ${ }^{12}$. En población alemana, el aumento del peso y de la grasa corporal no se asocia a una aparición más temprana pero si a un tiempo más corto de pubertad, en ambos sexos ${ }^{32}$.

Cabe señalar que hay diferencias entre los países, en la tendencia del tiempo en que se completan los eventos puberales. Factores nutricionales y ambientales, muchos de ellos productos químicos reconocidos como "disruptores endocrinos", podrían estar influyendo en estas diferencias, ya que algunos de ellos estimularían señales puberales del eje hipotálamo-hipófisisgónada y otros las inhibirían ${ }^{9,33,29}$. En los EEUU entre los años 1940 y 1994, hubo un significativo adelanto en la edad de inicio puberal con una estabilización en la edad de la menarquia, por lo que la duración de la pubertad es mayor ${ }^{4}$. En cambio en países europeos como Suecia y Alemania, el adelanto puberal se asocia a un menor tiempo de pubertad ${ }^{2,6,32,34}$. En nuestro estudio, se confirma un mayor tiempo de pubertad en ambos sexos. La m-2001 muestra un menor promedio de edad que la m-1986 en la etapa 2 y 3 de Tanner, sin diferencias en las etapas 4 y 5 . Por otro lado, el 
adelanto de la menarquia es muy discreto (12 años 3 meses y 12 años 1 mes respectivamente), lo que se traduce en un mayor tiempo entre la telarquia y la menarquia en la generación actual. Diferentes señales metabólicas que incluyen leptina, insulina y glucosa, modulan el eje hipotálamo-hipófisisgónada, postulándose varias hipótesis que asocian el sobrepeso con la pubertad temprana ${ }^{2}$. Una de ellas señala que la mayor secreción de leptina, secundaria al aumento de la grasa corporal, sería responsable de una estimulación más temprana del gonadostato hipotalámico ${ }^{35}$. Otra, sugiere que el hiperinsulinismo, asociado al aumento de la grasa corporal, estimularía la producción de andrógenos suprarrenales y estos activarían el gonadostato ${ }^{10}$. Ambas hipótesis explicarían también el mayor "tiempo" de pubertad, debido a que la activación del gonadostato, que inicia el proceso puberal, es un evento independiente de otras señales que dan cuenta del progreso de la pubertad ${ }^{2,9}$.

Finalmente, hay un dimorfismo sexual en la estatura. Las mujeres de la m-2001 parten su pubertad con menor estatura y llegan a la etapa 5 de Tanner con estaturas similares a las de la m-1986. En cambio los varones de la m-2001, parten su pubertad y la completan con estaturas significativamente mayores a la m-1986. Valenzuela y cols (1983) demostraron un menor crecimiento puberal en niñas de NSE medio-bajo, al compararlas con inglesas y francesas y un dimorfismo sexual en su talla final respecto al varón, sugiriendo determinantes étnicos para explicar ambos hechos ${ }^{36,37}$. Descartan un déficit ambiental selectivo para las mujeres y sugieren que estos hallazgos evidencian la presencia de genes para la estatura en los cromosomas sexuales $^{36}$. Por razones históricas asociadas al exterminio del varón mapuche y a la formación de parejas entre mujeres mapuches y varones españoles, habría un mayor mestizaje en los cromosomas $\mathrm{X}^{37}$.

Es importante reconocer algunas debilidades de este trabajo. Fue realizado en escolares de establecimientos subvencionados de la RM, por lo que estaría representando a esta región y a ese tipo de establecimientos. Esta forma de selección se hizo para hacer comparables ambas muestras en el NSE y representativa del grupo más numeroso, que es el medio. Los niveles socioeconómicos medios constituyen el grupo más numeroso en los establecimientos subvencionados tanto en la década del 80 como en la actualidad ${ }^{15,16,38}$. Por otra parte, el
Compendio de Información del MINEDUC 2002 señala que $52 \%$ de los escolares están en la RM.

Concluimos que durante la etapa de posttransición se observa una tendencia a una pubertad más temprana en escolares de ambos sexos, asociada a una mayor duración. Posiblemente esta tendencia tiene un determinante de tipo nutricional debido al aumento significativo del sobrepeso en este período; sin embargo, no pueden descartarse otros disruptores ambientales ${ }^{29,33}$. El dimorfismo sexual de la estatura apoyaría la hipótesis de Valenzuela y cols, quienes plantean que $95 \%$ de la población chilena se habría originado de la mezcla de varones españoles y mujeres aborígenes $^{36,37}$. Sería importante efectuar estudios similares en escolares de los NSE alto y bajo, dado que factores étnicos y ambientales (sobrepeso, dieta y actividad física), que influencian la pubertad, muestran diferencias por $\mathrm{NSE}^{26,38}$.

\section{Referencias}

1. Ebling FJ. The neuroendocrine timing of puberty. Reproduction 2005; 129: 675-83.

2. Parent AN, Teilmann G, Juul A, Skakkebaek NE, Toppari J, Bourguignon J. The timing of normal puberty and the age limits of sexual precocity: Variation around the world, secular trends and changes after migrations. Endocrine Rev 2003; 24: 668-93.

3. Delemarre-van WH. Secular trends of timing of puberty. Endocr Dev 2005; 8: 1-14.

4. Eulling SY, Herman-Giddens ME, Lee PA, Selevan SG, Juul A, Sorensen TA, et al. Examination of US Pubertytiming data from 1940 to 1994 for secular trends: Panel Findings. Paediatrics 2008; 121: 172-91.

5. Cole TJ. The secular trend in human physical growth: a biological view. Economics and Human Biology 2003; 1: 161-8.

6. Muinck Keizer-Schrama, Mul D. Trends in pubertal development in Europe. Human Reprod Upd 2001; 7: 287-91.

7. Rogol AD, Clark PA, Roemmich JN. Growth and puberal development in children and adolescent: effects of diet and physical activity. Am J Clin Nutr 2000; 72: 521-8.

8. Anderson SE., Dallal GE., Must A. Relative weight and race influence average age at menarche: results from two national representative surveys of US girls studied 25 years apart. Pediatrics 2003; 111: 844-50.

9. Buck GM, Gray LE, Marcus M, Ojeda S, Pescovitz OH, Feldman Witchel S, Spell W et al. Enviromental factors and timing puberty: Expert panel research needs. Pediatrics 2008; 121: 192-207. 
10. Kaplowitz PB, Slora EJ, Wasserman RC, Pedlow SE, Herman-Giddens ME. Earlier onset of puberty in girls: Relation to increased Body Mass Index and race. Pediatrics 2005; 108: 347-53.

11. Herman-Giddens ME, Slora EJ, Wasserman RC, Bourdony CJ, Bhapkar MV, Koch GG, Hasemeier CM. Secondary sexual characteristics and menses in young girls seen in office practice: a study from the Pediatric Research in Office setting network. Pediatrics 1997; 99: 505-12.

12. Wang Y. Is obesity associated with early sexual maturation? A comparison of the Association in American boys versus girls. Pediatrics 2002; 110: 903-10.

13. Díaz E. Breves observaciones sobre la aparición de la pubertad en la mujer chilena y de las predisposiciones patológicas propias del sexo. Memoria para optar al grado de Licenciado en Medicina. Santiago de Chile. 1988. Imprenta Nacional. DIBAM, MC0004516.

14. Avendaño A, Patrí A, Valenzuela C. Antropometría de escolares Fiscales del Área Norte de Stgo. Tercera parte: Maduración sexual. Cuad Med Soc 1975; 16: 17-24.

15. Burrows R, Leiva L, Mauricci A, Zvaigghaft A, Muzzo S. Características de la pubertad de niñas escolares de la Región Metropolitana. Rev Chil Pediatr 1988; 59: 21-5.

16. Muzzo S, Leiva L, Burrows R. Características antropométricas de escolares en el momento de la menarquia de acuerdo a la edad, NSE y área geográfica. Rev Chil Nutr 1994; 22: 144-9.

17. Gaete X, Unanue N, Avila A, Cassorla F. Cambios en la edad de inicio de la pubertad en niñas de la comuna de Santiago: implicancias para el diagnóstico de la pubertad precoz. Rev Chil Pediatr 2002; 73: 363-8.

18. Codner E, Unanue N, Gaete X, Barrera A, MookCanamori D, Bazaes R y cols. Cronología del desarrollo puberal en niñas escolares de Santiago: relación con NSE e índice de masa corporal. Rev Med Chile 2004; 132: 801-8.

19. Hernández MI, Unanue N, Gaete X, Cassorla F, Codner E. Edad de la menarquia y su relación con el nivel socioeconómico e índice de masa corporal. Rev Med Chile 2007; 135: 1429-36.

20. Muzzo S, Burrows R, Cordero J, Ramírez I. Trends in nutritional status and stature among school-age children in Chile. Nutrition 2004; 20: 867-72.

21. Kain J, Burrows R, Uauy R. Obesity trends in children and adolescents: Basic Determinants. In: Obesity in childhood and adolescence. Ed. Chen Ch. Ans Dietz W. Beijing, China. Agosto 2001. Nestlé Nutrition Workshop Series. Vol 49. pp 45-62.

22. Vio F, Albala C. Obesidad en Chile: una mirada epidemiológica. En Albala C, Kain J, Burrows R, Díaz E. editores. Obesidad un desafío pendiente. Santiago: Editorial Universitaria 2000: 31-43.
23. Marshall W., Tanner J. Variations in pattern of pubertal changes in girls. Arch Dis Child 1969; 44: 291-303.

24. Marshall W, Tanner J. Variations in pattern of pubertal changes in boys. Arch Dis Child 1970; 45: 13-23.

25. Unidad de Nutrición MINSAL, Consejo asesor de nutrición y Grupo de expertos. Norma técnica de evaluación nutricional del niño de 6 a 18 años. Reporte Técnico. Rev Chil Nutr 2004; 31: 128-37.

26. Olivares S, Kain J, Lera L, Pizarro F, Vio F, Moron C. Nutritional status, food consumption and physical activity among chilean school children. A descriptive study. Eu J Clin Nutr 2004; 58: 1278-85.

27. Berríos X. Tendencia temporal de los factores de riesgo de enfermedades crónicas: la antesala silenciosa de una epidemia que viene. Rev Med Chile 1997; 125: 1405-7.

28. Golub MS, Collman GW, Foster PD, Kimmel CA, Rajpert-De-Meyts E, Reiter EO et al. Public health implications of altered puberty timing.Pediatrics 2008; 121: 218-29.

29. Schoeters G, Den Hond E, Dhooge W, van Larebecke N Leijs M. Endocrin disruptors and abnormalities of pubertal development. Basic \& Clin Pharma \& Toxicology 2007; 102: 168-75.

30. Kranhnstoever K, Susman EJ, Lipps L. Percent body fat at age 5 predicts earlier puberal development among girls at age 9. Pediatrics 2003; 111: 815-21.

31. Kaplowitz PB. Link between body fat and the timing of puberty. Pediatrics 2008; 121: 208-17.

32. Buyken AE, Karaolis-Donckert N, Remer T. Association of prepuberal body composition in healthy girls and boys with the timing of early and late puberal markers. Am J Clin Nutr 2009; 89: 221-30.

33. Howdeshell KL, Hotchkiss AK, Thayer KA, Vanderbergh JG, Von Saal FS. Exposure to bisphenol A advances puberty. Nature 1999; 401: 763-4.

34. Hauspie RC, Vercauteren M, Susanne C. Secular changes in growth and maturation: an update. Acta Paediatr (Suppl 1) 1997; 423: 20-7.

35. Clement K, Vaisse C, Lahlan N, et al. A mutation in the human leptin receptor gene causes obesity and pituitary dysfunction. Nature 1998; 392: 398-401.

36. Valenzuela CY. Dimorfismo sexual pondoestatural en una población chilena ¿Evidencias de genes para la estatura en los cromosomas sexuales? Rev Med Chile 1975; 103: 322-5.

37. Valenzuela CY. Puberal origin of the larger sex dimorphism for the adult stature of a Chilean population. Am J Phys Anthropol 1983; 60: 53-60.

38. Burrows R, Montoya A, Gattas V, Díaz E, Sciaraffia $\mathrm{V}$, Lera L. Hábitos de ingesta y actividad física en escolares de enseñanza básica y media según tipo de establecimiento al que asisten. Rev Med Chile 2008; 136: 53-63. 